\title{
Remotely Sensing Image Fusion Based on Wavelet Transform and Human Vision System
}

\author{
Hui Lin ${ }^{1,2, a}$,Yanfeng Tian ${ }^{3, b} \quad$ Ruiliang $\mathrm{Pu}^{2, \mathrm{c}}$ and Liang Liang ${ }^{1, \mathrm{~d}}$ \\ ${ }^{1}$ School of Geodesy and Geomatics, Jiangsu Normal University, Xuzhou, Jiangsu, \\ 221116, P.R.China \\ ${ }^{2}$ Department of Geography, University of South Florida, Tampa, FL, 33620, USA \\ ${ }^{3}$ School of Environment Science and Spatial Informatics, China University of Mini \\ ng and Technology, Xuzhou, Jiangsu 221116, P.R.China \\ alinhuixznu@126.com, ${ }^{b} 505231462 @ q q . c o m,{ }^{c}$ rpu@usf.edu, ${ }^{d}$ liangl@jsnu.edu.cn
}

\begin{abstract}
Wavelet transform has many good characteristics, used extensively in image fusion. In recent years, many algorithms have been developed, but there exist some inherent defects such as image blur, burr phenomenon, zigzag boundaries and image discontinuity. In this theory, without considering disadvantages of HVS, especially fused image should preserve brightness and texture features which are the most sensitive to eye, so a new algorithm combining them is proposed. Firstly, by calculating brightness and texture metrics in different wavelet decomposition subimages. And then, by using root mean square rule to get fused low frequency and high frequency coefficients respectively. Finally, performing inverse wavelet transform by the concatenation of low frequency and high frequency to gain fused image. In order to evaluate different algorithms, the assessment metric based on HVS is adopted, which is a more comprehensive and effective measure. Experiments merging IKONOS Pan image(resolution is 1 meter) with multispectral image (resolution is 4 meter) show that the proposed algorithm is the best on brightness, contrast, texture, definition, resolution, object edge regardness of visual effect and objective metric, also verifing human visual characteristic to be considered in image fusion.
\end{abstract}

Keywords: image fusion; wavelet transform; human visual characteristics; brightness metric; texture metric; root mean square rule

\section{Introduction}

Image fusion is to use a specific algorithm integrated two or more images into a new image, which has a more comprehensive and clear representation about the same scene because the fused image can enough consider spatial-temporal correlation and complementary among them [7]. Due to the characteristics of multi-scale analysis and localization in both time and frequency domain, wavelet transform is regarded as "mathematics magnifying glass" in signal analysis and processing [9]. The characteristic of multiresolution analysis is similar to human vision model, meanwhile, wavelet decomposition is directional, which coincides with the vision feature that human has different resolution on different high frequency subimages; fused image using wavelet transform can gain better visual effect. So it is used extensively in image fusion, developed many algorithms gradually, such as maximum coefficients, weighed averaging, local energy rule and variance-covariance rule $[1,6]$, which do not take human vision system into consideration, resulting in burrs in local image, ring effect due to the loss of some edge information of original image, image continuity damage owing to different fusion rule about low frequency and high frequency Subimages [4]. A new fusion 
algorithm based on Human Vision System(HVS) is proposed, which firstly figure out brightness and texture measure of each pixel neighborhood on every wavelet decomposition scale [5], and then, getting the weighed fusion coefficients adaptively. Furthermore, adopting hierarchical iterative to perform inverse wavelet transform to generate the fused image. to evaluate the fused image, the new assessment metric combining objective and subjective factor is presented. Experiments show that the proposed method can not only improve image fusion effect, but also be favorable for target detection and recognition.

\section{Proposed Method}

\subsection{Wavelet based Image Fusion}

Because wavelet transform has good localization characteristics in frequency domain and time domain, it is widely used in image processing and analysis.

Mallat proposed fast wavelet decomposition algorithm and reconstruction algorithm, which used two one-dimensional filter to realize two-dimensional images decomposition, next, he used two 2D filters to conduct image reconstruction [11].

If low pass $(H)$ and high pass $(G)$ are two one dimensional mirror image filtering operators, the subscript $r, c$ corresponds to image rows and column respectively, according to the Mallat algorithm, we have the following decomposition formula in the scale of $j-1$ :

$$
\left\{\begin{array}{l}
C_{j}=H_{c} H_{r} C_{j-1} \\
D_{j}^{v}=G_{c} H_{r} C_{j-1} \\
D_{j}^{h}=H_{c} G_{r} C_{j-1} \\
D_{j}^{d}=G_{c} G_{r} C_{j-1}
\end{array}\right.
$$

The reconstruction algorithm is expressed as follows:

$$
C_{j-1}=H_{r}^{*} H_{c}^{*} C_{j}+H_{r}^{*} G_{c}^{*} D_{j}^{v}+G_{r}^{*} H_{c}^{*} D_{j}^{h}+G_{r}^{*} G_{c}^{*} D_{j}^{d}
$$

where $H^{*} 、 G^{*}$ are the conjugate transpose matrixes of $H 、 G, D_{j}^{i}$ and $C_{j-1}$ is wavelet coefficient and scale coefficient in the scale of $j(i=v, h, d)$ respectively, $D_{j}^{v}$ 、 $D_{j}^{h} 、 D_{j}^{d}$ indicate high frequency of vertical, horizontal, diagonal correspondingly. Fig.1 illustrates the flow block diagram for image fusion using wavelet transform, the key is fusion rule on wavelet decomposition coefficient.

\subsection{Wavelet based Image Fusion Considering HVS}

HVS is used to study the theory of human visual characteristics. In the field of image processing, it specializes in the problem that which areas in image is more sensitive and interested to human vision. HVS analysis in wavelet domain refers to the analyzing human visual characteristics, and different weighs are given different region of decomposition subimages. Brightness feature and texture feature are most sensitive image characteristics for eye from the point of view of human visual characteristics, theoretically, if the two features are taken into account that the fused image can fully retain a majority of texture and sketch information included in original images [10]. 


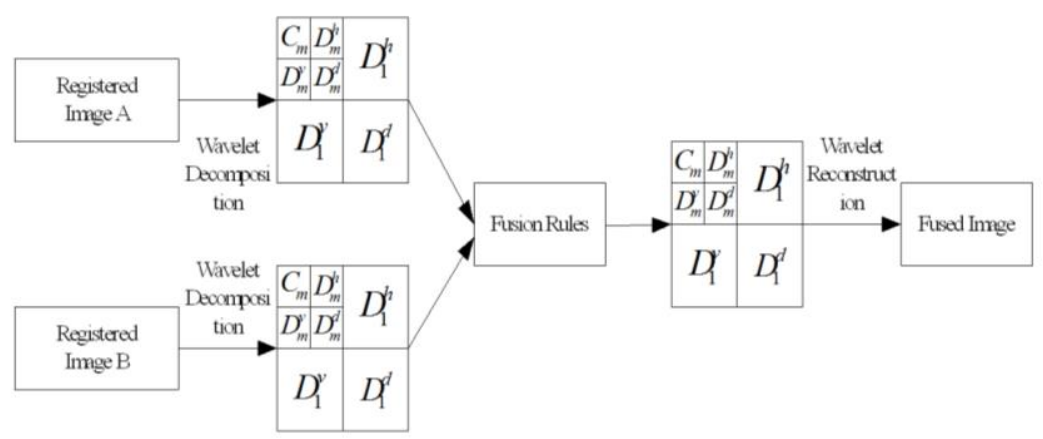

Figure 1. Wavelet based Image Fusion Framework

So image fusion algorithm integrating ilbrightness and texture features is proposed, which describes edge, texture, high ilbrightness area by visual characteristic model, and calculating weigh coefficient adaptively, finally adopting weighed averaging to reconstruct fused image.

Quantitative estimates of brightness and texture features show by the following expression [10]:

$$
\begin{aligned}
L(m, x, y)= & +\frac{1}{256} \sum_{i=0}^{l} \sum_{j=0}^{l} I^{3, L L}\left(i+1+\frac{x}{2^{3-r}}, j+1+\frac{y}{2^{3-r}}\right) \\
T(r, x, y) & =\sum_{k=1}^{3-r} 16^{-k} \sum_{S}^{L H, H H, H L} \sum_{i=0}^{l} \sum_{j=0}^{l}\left(I^{k+r, s}\left(i+\frac{x}{2^{k}}, j+\frac{y}{2^{k}}\right)\right)^{2}, \\
& +16^{3-r} \operatorname{var}\left(I^{3, L L}\left(\{1,2\}+\frac{x}{2^{3-r}},\{1,2\}+\frac{y}{2^{3-r}}\right)\right)
\end{aligned},
$$

where $r$ is the number of levels of wavelet decomposition, $(x, y)$ is the pixel coordinate, superscript $k$ is the scale of wavelet decomposition, which represents non-low frequency coefficient of certain decomposition level.

Function $L$ is used to calculate the brightness value of the edge of each pixel $(x, y)$, which indicates edge brightness change of pixel $(x, y)$ neighborhood in high frequency domain. Similarly, function $T$ is used to calculate the texture information included in each pixel $(x, y)$, which involves low frequency coefficient in each subimage.

\subsection{Fusion Rule based on HVS}

HVS fusion algorithm based on brightness and texture measure is introduced briefly as follows:

1) Preprocess the original image, get registered image with same resolution in the same area, and perform wavelet transform to get the multi-scale representation of each source image;

2) On each scale, use HVS measure to express target of interest in every subimage respectively;

3) Through the HVS metric to calculate the weighted coefficient of different frequencies;

4) Complete fusion in the wavelet domain, and through wavelet reconstruction to get the fused image.

Among steps, step 3 is the most important. In fact, it is a fusion rule. The calculation of weighted coefficient considering brightness and texture features is divided into two parts, one is low frequency, the other is high frequency, and the specific procedure is as follows:

(1) The weighted fusion of low frequency coefficient 


$$
\begin{aligned}
& \text { Let } W_{\max }^{L L}(x, y)=1-\frac{L_{A}(x, y) \cdot L_{B}(x, y)}{L_{A}^{2}(x, y)+L^{2}(x, y)}, W_{\min }^{L L}(x, y)=1-W_{\max }^{L L}(x, y), \\
& \text { If } L_{A}(x, y)>L_{B}(x, y), \text { then we can get fused low frequency coefficient : } \\
& \qquad I^{L L}(x, y)=I_{A}(x, y) * W_{\max }^{L L}(x, y)+I_{B}(x, y) * W_{\min }^{L L}(x, y)
\end{aligned}
$$

If $L_{A}(x, y)<L_{B}(x, y)$, then we can get fused low frequency coefficient :

$$
I^{L L}(x, y)=I_{B}(x, y) * W_{\max }^{L L}(x, y)+I_{A}(x, y) * W_{\min }^{L L}(x, y)
$$

Where the subscript $A, B$ is two registered original images, $I_{A}(x, y)$ and $I_{B}(x, y)$ denote low frequency values of pixel $(x, y)$ in image $A, B . L_{A}(x, y), L_{B}(x, y)$ is the brightness feature value of pixel $(x, y)$ in image $A, B$, the above expression indicates weight value change with the brightness of original images, so the brightness sensitive to human vision is fully taken into account.

(2) The weighted fusion of high frequency coefficient

$$
\begin{aligned}
& \text { Let } W_{\max }^{H}(x, y)=1-\frac{T_{A}(x, y) \cdot T_{B}(x, y)}{T^{2}{ }_{A}(x, y)+T^{2}{ }_{B}(x, y)} \quad W_{\min }^{H}(x, y)=1-W_{\max }^{H}(x, y), \\
& \text { If } T_{A}(x, y)>T_{B}(x, y) \text {, then we can get fused high frequency coefficient: } \\
& \qquad I^{H}(x, y)=I_{A}(x, y) * W_{\max }^{H}(x, y)+I_{B}(x, y) * W_{\min }^{H}(x, y) \\
& \text { If } T_{A}(x, y)<T_{B}(x, y) \text {, then we can get fused high frequency coefficient: } \\
& \qquad I^{H}(x, y)=I_{B}(x, y) * W_{\max }^{H}(x, y)+I_{A}(x, y) * W_{\min }^{H}(x, y)
\end{aligned}
$$

In the course of weighted coefficient calculation, the mean square criterion is adopted, which can prevent the expansion of image reconstruction errors.

At the same time, if brightness and texture measure is larger, weighted coefficient is given larger in order to better highlight the characteristics of the original image.

After subimage fusion in different scales, the final step is to put all fused coefficient together to perform inverse wavelet transform to reconstruct fused image.

\subsection{Evaluation Metric of Fused Image}

Evaluation of image fusion result can be divided into two categories separately, namely, subjective evaluation and objective evaluation. The advantage of the former is simple, perceptual intuition, but a lot of subjective factors affect the evaluation results.

Traditional objective evaluation is mainly decided by many metrics such as mean value, variance, standard deviation, average gradient, cross entropy and mutual information [13]. Although these metrics are objective, independent, their theory bases on error between pixels or statistical characteristic of image, which results in incorrect evaluation, the reason is that human eye visual feature is adquately considered.

Among the above metrics, mean value, standard deviation and entropy aim to a single image evaluation, mainly reflecting the absolute quality of an image, especially can't reflect the fused image's relationship with the source image. Root of mean square and cross entropy are needed ideal image, but unable to get an ideal image in the practical application [12], in addition, in many image applications, the end user is a person. Owing to not considering human eyes visual characteristics, the evaluation results are often inconsistent with subjective visual effect. So an evaluation method combining subjective with objective factor is adopted.

Assume that the sizes of image $A, B$ are $M \times N, \bar{A}, \bar{B}$ denote their mean value, $\alpha_{A}^{2}, \alpha_{B}^{2}$ indicate their variance, $\alpha_{A}, \alpha_{B}$ are their standard deviation, $E_{A}, E_{B}$ are their entropy, $p_{i A}, p_{i B}$ are the probability of gray value $i$ in image $A, B$. Expression is below: 


$$
\begin{gathered}
\bar{A}=\frac{1}{M N} \sum_{x=1}^{M} \sum_{y=1}^{N} A(x, y), \quad \bar{B}=\frac{1}{M N} \sum_{x=1}^{M} \sum_{y=1}^{N} B(x, y) \\
\alpha_{A}^{2}=\frac{1}{M N} \sum_{x=1}^{M} \sum_{y=1}^{N}(A(x, y)-\bar{A})^{2}, \alpha_{B}^{2}=\frac{1}{M N} \sum_{x=1}^{M} \sum_{y=1}^{N}(B(x, y)-\bar{B})^{2} \\
E_{A}=-\sum_{i=1}^{m} p_{i A} \ln p_{i A}, E_{B}=-\sum_{i=1}^{m} p_{i B} \ln p_{i B}
\end{gathered}
$$

Then we may define:

$$
Q(A, B)=\frac{2 \alpha_{A} \alpha_{B}}{\alpha_{A}{ }^{2}+\alpha_{B}{ }^{2}} \cdot \frac{2 \bar{A} \bar{B}}{\bar{A}^{2}+\bar{B}^{2}} \cdot \frac{E_{A}}{E_{B}}
$$

In image fusion, the fused image is generally obtained by two original images, so the image fusion quality evaluation must comprehensively consider the relations between source images and fused image. We construct function $Q(F, A, B)$,

let

$$
Q(F, A, B)=\lambda Q(F, A)+(1-\lambda) Q(F, B) .
$$

Where $A, B$ are two source images, $F$ is fused image, $\lambda$ represents weighted coefficient, $\lambda \in[0,1]$, in general, $\lambda=0.5$. of course, if one image is more important than the other, the larger $\lambda$ may be gotten, in theory, $Q(F, A, B) \in[0,2]$, if the value is larger, the fusion effect is better.

\section{Experiments and Analysis}

Image fusion experiment is performed to verify the algorithm proposed in this paper. For the comparison's sake, Wavelet based weighted fusion and discrete wavelet transform are selected, the IKONOS PAN image and multispectral images of downtown area on November 18, 2010 are experimental data, whose resolutions are 1 meter and 4 meter respectively, the original registered images and fused images see Fig.1, the purpose of fusion is to preserve the high spatial resolution of PAN image and the high spectral resolution of multispectral image.

Through visual effect, fused images by all means are better than original image in spatial resolution, definition, objects feature and spectral retaining, which are rich in texture information. In comparison, Fig.1(e) has better visual effect than the other two methods, furthermore, Fig.1(c) and Fig.1(d) are blur, object boundaries such as house, road exist burrs and zigzag edge. The results show that the fusion image without considering human vision system is not ideal, the difference is obvious.

Image quality evaluation under different fusion algorithms is performed by adopting new $Q$ metric, the values are shown in the table 1 , where $\lambda=E_{A} / E_{B}$ or $\lambda=E_{B} / E_{A}$

, $A, B, F$ represent PAN image, multispectral image and fused image. $Q(F, A, B)$ is the total assessment between the two original images and fused image, $Q(F, A)$ is the assessment between the PAN image $A$ and fused image $F, Q(F, B)$ is the assessment between the multispectral image $B$ and fused image $F$. From the table, we know that $Q(F, A, B), Q(F, A)$ and $Q(F, B)$ values are more than 1, which illustrate the fact that fused image $F$ is better than other original images $A, B$ in brightness, contrast, entropy, edge and object feature, in particular, the proposed algorithm $(Q(F, A, B)=1.3879)$ considering brightness and texture measure of HVS is prior to others. Meanwhile, the results are consistent with visual effect fully. 


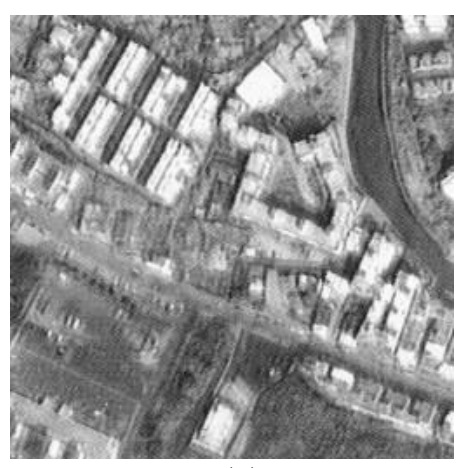

(a)

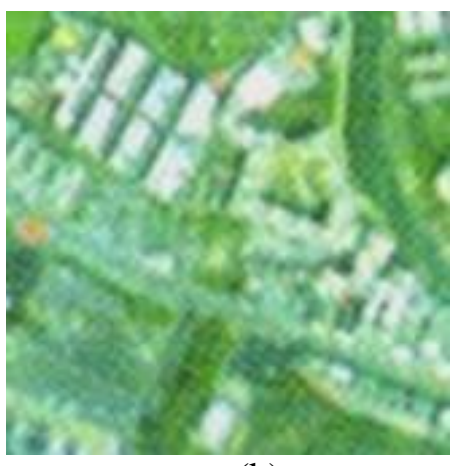

(b)

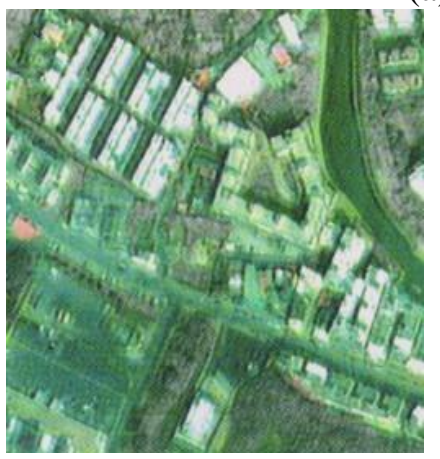

(c)

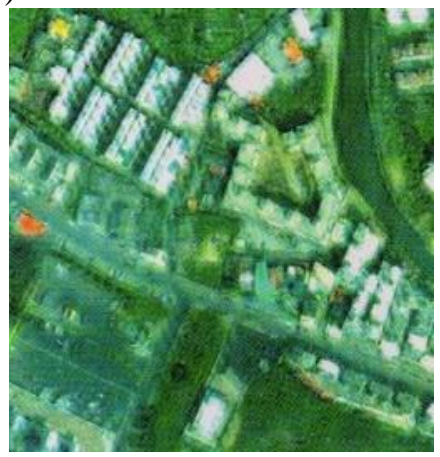

(d)

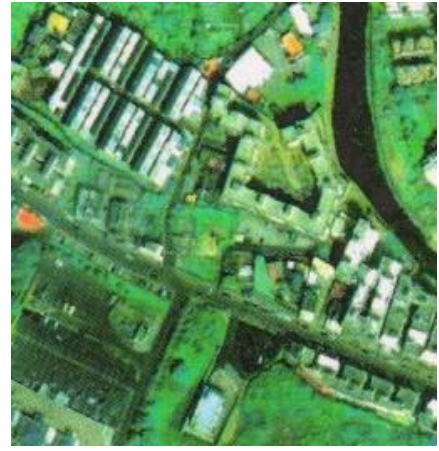

(e)

Figure 2. Original registered images and fused images using different algorithm: (a) IKONOS PAN image,(b)IKONOS multispectral image ,(c) discrete 2D wavelet transform fusion, (d) Wavelet based weighted fusion,(e)the proposed method

Table 1. Image Evaluation under Different Fusion Algorithms

\begin{tabular}{|c|c|c|c|}
\hline Fusion algorithm & $Q(F, A, B)$ & $Q(F, A)$ & $Q(F, B)$ \\
\hline $\begin{array}{l}\text { discrete 2D wavelet } \\
\text { transform fusion }\end{array}$ & 1.1963 & 1.1698 & 1.2140 \\
\hline $\begin{array}{l}\text { Wavelet based } \\
\text { weighted fusion }\end{array}$ & 1.3104 & 1.2445 & 1.3543 \\
\hline $\begin{array}{l}\text { The proposed } \\
\text { algorithm }\end{array}$ & 1.3879 & 1.2665 & 1.4689 \\
\hline
\end{tabular}

\section{Conclusions}

Based on wavelet transform image fusion has made great progress, many algorithms have been developed. There exist two serious problems: one is fusion rule, which without thinking about HVS, resulting in burrs and zigzag edge emerging, the loss of edge information and the destroyed continuity between low frequency and high frequency. In view of defects of existing wavelet based methods, we consider human vision system, a new algorithm which combines HVS with wavelet transform is proposed, and mainly introduce the most sensitive human visual features such as brightness and texture measure in fusion rule. Another is image quality evaluation criterion, which need to be established urgently. In the analysis of existing evaluation, a more comprehensive evaluation measure integrating subjective with objective factors is presented. At that time, an experiment image fusion between high resolution PAN image and multispectral image is performed under three different algorithms. Evaluation results indicate the proposed algorithm is very practical and effective. The objective evaluation is consistent with subjective visual 
effect adequately, HVS based image fusion is future development tendency. But how to integrate human visual characteristics into different fusion rules is still of vital [13].

\section{Acknowledgements}

This work was financially supported by, Natural Science Foundation (411713213, 41401473 ) , Funded by Key Laboratory of Geo-informatics of State Bureau of Surveying and Mapping) (TJES1204), Funded by Key Laboratory of Advanced Engineering Surveying of State Bureau of Surveying and Mapping (201310) .

\section{References}

[1] A. Wang, H. Sun and Y. Guan, "The application of wavelet transform to multimodality medical image fusion", Proc. IEEE International Conference on Networking, Sensing and Control (ICNSC), Ft.Lauderdale, Florida, (2006), pp. 270-274.

[2] C. Yang, J. Q. Zhang, X. R. Wang and X. Liu, „A novel similarity based quality metric for image fusion", Information Fusion, vol. 9, (2008), pp. 156-160.

[3] H. Chen and P. K. Varshney, "A human perception inspired quality metric for image fusion based on regional information", Information Fusion, vol. 8, (2007), pp. 193-207.

[4] H. Zhang, L. Liu and N. Lin, "A novel wavelet medical image fusion method", International Conference on Multimedia and Ubiquitous Engineering (MUE’07), Seoul, Korea, (2007), April, pp. 114-118.

[5] J. Gao, Z. Liu and T. Ren, "A new image fusion scheme based on wavelet transform", Proc., 3rd International Conference on Innovative Computing,Information and Control, Dalian, China, (2008) June, pp. 423-428.

[6] J. J. Lewis, R. J. O_Callaghan, S. G. Nikolov, D. R. Bull and C. N. Canagarajah, "Region-based image fusion using complex wavelet, The Seventh International Conference on Image Fusion, Stockholm, (2004), pp. 524-529.

[7] L. Chipman, T. Orr and L. Graham, "Wavelets and image fusion", Wavelet Applications in Signal and Image Processing III, vol. 2569, (1995), pp. 208-219.

[8] S. Li, J. T. Kwok and Y. Wang, "Using The Discrete Wavelet Frame Transform To Merge Landsat TM And SPOT Panchromatic Images”, Information Fusion, vol. 3, (2002), pp. 17-24.

[9] O. Rockinger, "Pixel-level fusion of image sequences using wavelet frames", Proc. of the 16th Leeds Applied Shape Research Workshop,Leeds University Press, (1996), pp. 149-154.

[10] P. R. Hill, "Wavelet Based Texture Analysis and Segmentation for Image Retrieval and Fusion", Ph.D. Thesis, Department of Electrical and Electronic Engineering, University of Bristol, UK, (2002).

[11] S. G. Mallat, "A theory for multiresolution signal decomposition: The wavelet representation", IEEE Trans. Pattern Anal. Mach. Intell., vol.11, (1989), pp. 674-693.

[12] V. Petrovic, "Subjective tests for image fusion evaluation and objective metric validation", Information Fusion, vol. 8, (2007), pp. 208-216.

[13] Z. Wang and A. C. Bovik, "A universal image quality index", IEEE Signal Processing Letter, vol. 9, no. 3, (2002), pp. 81-84.

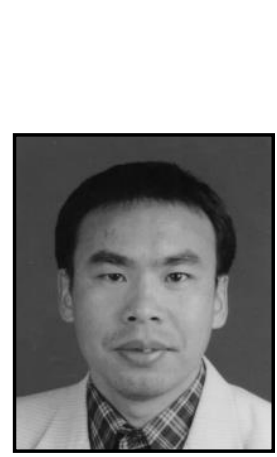

\section{Author}

Hui Lin, he was born in 1973, associate professor, from Hunan Province of China, working in Jiangsu Normal University, research interest: image processing of remote sensing. 
International Journal of Signal Processing, Image Processing and Pattern Recognition Vol.8, No.7 (2015) 